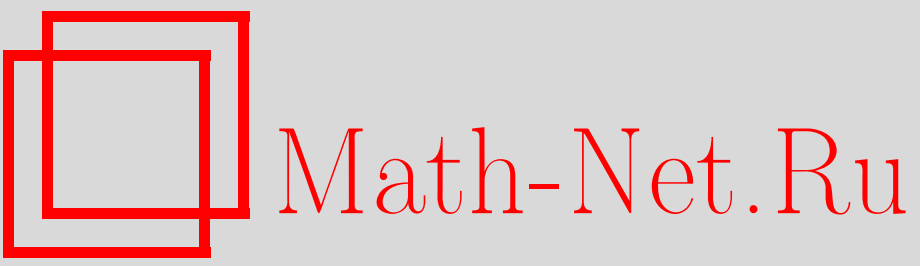

А. В. Разумов, Ю. Г. Строганов, О детальном перечислении некоторых классов симметрии матриц чередующихся знаков, ТMФ, 2004, том 141, номер 3, 323-347

DOI: https://doi.org/10.4213/tmf133

Использование Общероссийского математического портала Math-Net.Ru подразумевает, что вы прочитали и согласны с пользовательским соглашением

http://www . mathnet.ru/rus/agreement

Параметры загрузки:

IP: 3.85 .73 .92

26 апреля 2023 г., 14:24:09 
ТЕОРЕТИЧЕСКАЯ

И МАТЕМАТИЧЕСКАЯ

ФИЗИКА

Том 141, № 3

декабрь, 2004

(C) 2004 г.

А.В. Разумов*, Ю. Г. Строганов*

\section{О ДЕТАЛЬНОМ ПЕРЕЧИСЛЕНИИ НЕКОТОРЫХ КЛАССОВ СИММЕТРИИ МАТРИЦ ЧЕРЕДУЮЩИХСЯ ЗНАКОВ}

На основании детерминантных представлений для статистических сумм соответствующих вариантов моделей квадратного льда, а также метода, предложенного недавно одним из авторов, исследованы детальные перечисления вертикально симметричных матриц чередующихся знаков, внедиагонально симметричных матриц чередующихся знаков и матриц чередующихся знаков с возвратной границей. Для всех рассмотренных случаев найдены явные формулы для перечислений. В частности, доказана гипотеза Кутина-Юена.

Ключевые слова: матрицы чередующихся знаков, перечисления, модель квадратного льда.

\section{1. ВВЕДЕНИЕ}

Матрица чередующихся знаков - это матрица с элементами $1,0,-1$, такая что 1 и -1 чередуются в каждом столбце и в каждой строке и, кроме того, первый и последний ненулевые элементы в каждом столбце и в каждой строке равны 1. За последнее десятилетие было предложено и частично доказано много утверждений о перечислениях и о равенствах перечислений, относящихся к матрицам чередуюшихся знаков. Тем не менее остается много задач, ожидаюших решения. Помимо их важности для чистой комбинаторики, результаты, полученные для перечислений матриц чередующихся знаков, несомненно найдут многочисленные применения в задачах математической физики (см. в этой связи, например, работы [1]).

В настоящей статье, используя метод, предложенный недавно одним из авторов [2], мы исследуем детальные перечисления некоторых классов симметрии матриц чередующихся знаков. В разделе 2 воспроизведены необходимые результаты работы [2]. В разделе 3 найдены детальные перечисления матриц чередуюшихся знаков с возвратной границей и вертикально симметричных матрищ чередуюшихся знаков (см. ниже следствие 3$)$. В разделе 4 доказано, что детальные перечисления вертикально симметричных

\footnotetext{
* Институт физики высоких энергий, Протвино, Московская обл., Россия. E-mail: razumov@mx.ihep.su; stroganov@mx.ihep.su
} 
матриц чередующихся знаков и внедиагональных матриц чередующихся знаков совпадают. Это утверждение является содержанием гипотезы Кутина-Юена, представленной в письме [3], направленном Купербергом участникам форума “Домино”. В двух следуюших абзацах мы практически дословно цитируем это письмо.

Упомянутые выше классы симметрии матриц чередующихся знаков определяются следующим образом. Матрица чередующихся знаков называется вертикально симметричной, если она не меняется при отражении относительно вертикальной линии, делящей эту матрицу на две равные части. Заметим, что только матрицы чередующихся знаков нечетного порядка могут быть вертикально симметричными. Матрица чередующихся знаков называется внедиагонально симметричной, если она не меняется при отражении относительно ее главной диагонали, а сама эта диагональ состоит из нулей. В работе [4] Куперберг доказал, что число внедиагонально симметричных $(2 n \times 2 n)$-матриц чередующихся знаков совпадает с числом вертикально симметричных $((2 n+1) \times(2 n+$ 1))-матриц чередуюшихся знаков. Для проверки этого совпадения перечислений Кутин и Юен провели компьютерный эксперимент и обнаружили, что равенство сохраняется, даже если фиксировать позицию единицы в крайнем правом столбце. Заметим, что любая матрица чередуюшихся знаков имеет только одну единицу в крайнем правом столбце. Здесь необходимо напомнить, что в вертикально симметричной матрице чередующихся знаков эта единица не может стоять в самой верхней или в самой нижней строке, а во внедиагонально симметричной матрице чередуюшихся знаков она не может находиться в самой нижней строке, поэтому в обоих классах матрищ мы имеем $2 n-1$ допустимую позицию.

Для примера сравним вертикально симметричные матрицы чередующихся знаков порядка 5 (см. рис. $1^{1)}$ ) с внедиагонально симметричными матрицами чередующихся знаков порядка 4 (см. рис. 2). В обоих случаях числа Кутина-Юена суть 1, 1, 1.

$$
\left(\begin{array}{lllll}
0 & 0 & + & 0 & 0 \\
+ & 0 & - & 0 & + \\
0 & 0 & + & 0 & 0 \\
0 & + & - & + & 0 \\
0 & 0 & + & 0 & 0
\end{array}\right) \quad\left(\begin{array}{lllll}
0 & 0 & + & 0 & 0 \\
0 & + & - & + & 0 \\
+ & - & + & - & + \\
0 & + & - & + & 0 \\
0 & 0 & + & 0 & 0
\end{array}\right) \quad\left(\begin{array}{ccccc}
0 & 0 & + & 0 & 0 \\
0 & + & - & + & 0 \\
0 & 0 & + & 0 & 0 \\
+ & 0 & - & 0 & + \\
0 & 0 & + & 0 & 0
\end{array}\right)
$$

Рис. 1. Вертикально симметричные матрицы чередующихся знаков порядка 5 .

$$
\left(\begin{array}{cccc}
0 & 0 & 0 & + \\
0 & 0 & + & 0 \\
0 & + & 0 & 0 \\
+ & 0 & 0 & 0
\end{array}\right) \quad\left(\begin{array}{cccc}
0 & 0 & + & 0 \\
0 & 0 & 0 & + \\
+ & 0 & 0 & 0 \\
0 & + & 0 & 0
\end{array}\right) \quad\left(\begin{array}{cccc}
0 & + & 0 & 0 \\
+ & 0 & 0 & 0 \\
0 & 0 & 0 & + \\
0 & 0 & + & 0
\end{array}\right)
$$

Рис. 2. Внедиагонально симметричные матрицы чередующихся знаков порядка 4.

\footnotetext{
1) Как это сейчас принято для матриц чередуюшихся знаков, мы пишем + вместо 1 и - вместо - 1 .
} 
Наше исследование основьвается на взаимно однозначном соответствии между состояниями модели квадратного льда с подходяшими граничными условиями и матрицами чередуюшихся знаков. Рассмотрим такое подмножество вершин и ребер квадратной решетки, что каждая внутренняя вершина четырехвалентна и каждая граничная вершина одновалентна. Состояние соответствующей модели льда определяется такой ориентацией ребер, что каждая четырехвалентная вершина имеет два входящих ребра и два выходяших. Если ориентация одновалентных ребер фиксирована, то мы говорим, что заданы граничные условия для рассматриваемой модели льда. При изучении матриц чередующихся знаков важную роль играют так называемые граничные условия типа доменной стенки. Шаблон для таких состояний изображен на рис. 3 . Смысл меток $x_{i}$ и $y_{i}$ будет объяснен ниже. Этот тип граничных условий был впервые рассмотрен Корепиным [5].

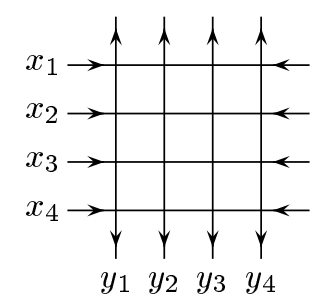

Рис. 3. Квадратный лед с граничными условиями типа доменной стенки.

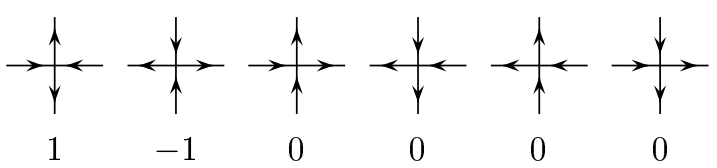

Рис. 4. Соответствие между вершинами квадратного льда и элементами матриц чередующихся знаков.

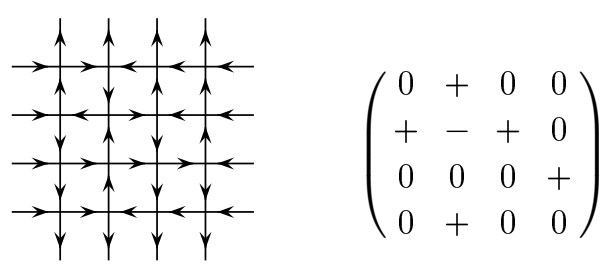

Рис. 5. Пример соответствия между состояниями квадратного льда и матрицами чередуюшихся знаков.

Если каждую четырехвалентную вершину, принадлежашую состоянию модели квадратного льда с граничными условиями типа доменной стенки, заменить числом согласно 
рис. 4 , то мы получим матрицу чередуюшихся знаков. Пример приведен на рис. 5. Нетрудно проверить, что таким образом мы получаем взаимно однозначное соответствие между состояниями и матрицами чередуюшихся знаков [6]. Впервые это соответствие было использовано для решения задач перечисления матриц чередующихся знаков Купербергом [7]. Его подход заключался в следующем. Рассмотрим статистическую сумму модели квадратного льда с граничными условиями типа доменной стенки. Эта сумма является суммой весов всех возможных состояний. Вес состояния определяется как произведение весов всех четырехвалентных вершин. Свяжем с горизонтальными линиями решетки спектральные параметры $x_{i}$, а с вертикальными линиями - спектральные параметры $y_{i}$ (см. рис. 3). Вершине, находящейся на пересечении линий со спектральными параметрами $x_{i}$ и $y_{j}$, приписывается спектральный параметр $x_{i} / y_{j}$. Веса вершин определяются значениями их спектральных параметров и приведены на рис. $6^{2)}$, где $a$ - общий для всех вершин параметр и использованы удобные обозначения $\bar{x}=x^{-1}$, $\sigma(x)=x-\bar{x}$, введенные Купербергом.

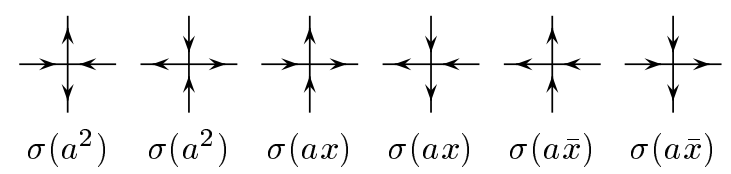

Рис. 6. Веса вершин со спектральным параметром $x$.

Обозначим статистическую сумму модели квадратного льда с граничными условиями типа доменной стенки как $Z(n ; \boldsymbol{x}, \boldsymbol{y})$. Здесь $n$ - размер квадратного льда, $\boldsymbol{x}$ и $\boldsymbol{y}$ - векторы, построенные из спектральных параметров: $\boldsymbol{x}=\left(x_{1}, \ldots, x_{n}\right), \boldsymbol{y}=\left(x_{1}, \ldots, x_{n}\right)$. Для заданного числа $x$ обозначим через $A(n ; x)$ полный вес $(n \times n)$-матриц чередующихся знаков, где вес индивидуальной матрицы чередующихся знаков принимается за $x^{k}$, если у нее $k$ матричных элементов равны -1 . Рассмотрим статистическую сумму $Z(n ; \boldsymbol{x}, \boldsymbol{y})$ при $\boldsymbol{x}=\mathbf{1}$ и $\boldsymbol{y}=\mathbf{1}$, где $\mathbf{1}=(1,1, \ldots, 1)$. Если число -1 в $(n \times n)$-матрице чередующихся знаков равно $k$, то число единиц равно $n+k$ и число нулей равно $n^{2}-n-2 k$. Ясно, что вес соответствующего состояния квадратного льда есть

$$
\sigma^{k}\left(a^{2}\right) \sigma^{n+k}\left(a^{2}\right) \sigma^{n^{2}-n-2 k}(a)=\sigma^{n^{2}-n}(a) \sigma^{n}\left(a^{2}\right)\left[\frac{\sigma\left(a^{2}\right)}{\sigma(a)}\right]^{2 k} .
$$

Следовательно, справедливо равенство

$$
A(n ; x)=\frac{1}{\sigma^{n^{2}-n}(a) \sigma^{n}\left(a^{2}\right)} Z(n ; \mathbf{1}, \mathbf{1}),
$$

где

$$
x=\left[\frac{\sigma\left(a^{2}\right)}{\sigma(a)}\right]^{2}=(a+\bar{a})^{2}
$$

${ }^{2)}$ В отличие от работы [2], здесь мы используем мультипликативные параметры. 
$\mathrm{B}$ частности, при $a=e^{i \pi / 3}$ имеем $x=1$, и соотношение (1) дает полное число $(n \times n)$ матриц чередуюшихся знаков:

$$
A(n)=\left.\frac{1}{\sigma^{n^{2}}(a)} Z(n ; \mathbf{1}, \mathbf{1})\right|_{a=e^{i \pi / 3}} .
$$

Таким образом, задача перечисления матриц чередующихся знаков сводится к изучению статистической суммы $Z(n ; \boldsymbol{x}, \boldsymbol{y})$ при $\boldsymbol{x}=\mathbf{1}$ и $\boldsymbol{y}=\mathbf{1}$.

В работе [7] Куперберг воспользовался представлением $Z(n ; \boldsymbol{x}, \boldsymbol{y})$ через детерминант Изергина-Корепина [8] и доказал формулу для $A(n)$, предложенную Миллсом, Робинсом и Рамсеем [9] и впервые доказанную Цайлбергером [10]. В работе [4] Куперберг обобшил детерминант Изергина-Корепина и детерминант Цучии [11] с целью исследования задач перечисления матриц чередуюшихся знаков, принадлежаших многочисленным классам симметрии.

В работе [12] Цайлбергер также использовал представление статистической суммы $Z(n ; \boldsymbol{x}, \boldsymbol{y})$ через детерминант Изергина-Корепина для доказательства так называемой гипотезы о детальном перечислении матриц чередуюшихся знаков, предложенной Миллсом, Робинсом и Рамсеем [9]. Объясним, в чем заключается смысл такого перечисления. Напомним, что первый столбец матрицы чередующихся знаков содержит только одну единицу, все остальные элементы равны нулю. Следовательно, мы можем попытаться пересчитать те матришы чередуюшихся знаков, у которых единственная единица первого столбца находится в позиции с номером $r$. Соответствующие числа $A(n, r)$ и являются решением задачи детального перечисления матриц чередующихся знаков. Как и для случая обычного перечисления, можно предположить, что вес отдельной матришы чередуюшихся знаков равен $x^{k}$, если она имеет $k$ матричных элементов, равных -1 . В результате получаем набор полиномов $A(n, r ; x)$, описьвающих взвешенное детальное перечисление матриц чередующихся знаков.

Для того чтобы связать полиномы $A(n, r ; x)$ со статистической суммой $Z(n ; \boldsymbol{x}, \boldsymbol{y})$, рассмотрим случай, в котором $\boldsymbol{x}=\mathbf{1}$ и $\boldsymbol{y}=(u, 1, \ldots, 1)$. В этом случае метка вершины, принадлежащей к первому столбцу, равна $u$, в остальных случаях она равна 1 . Рассмотрим состояние квадратного льда, у которого единственная единица первого столбца находится в $r$-й строке. При этом в первой строке имеется одна вершина первого типа, $r-1$ вершина третьего типа и $n-r$ вершин шестого типа, где тип определяется положением вершины на рис. 6 . Следовательно, вклад первого столбца в вес состояния равен $\sigma\left(a^{2}\right) \sigma^{r-1}(a \bar{u}) \sigma^{n-r}(a u)$. Обозначим через $k$ число элементов, равных -1 , в рассматриваемой матрице чередуюшихся знаков. Тогда число единиц в этой матрице чередующихся знаков без первого столбца равно $n-1+k$, а число нулей равно $n^{2}-2 n+1-2 k$. Таким образом, вес рассматриваемого состояния есть

$$
\sigma^{n^{2}-2 n+1}(a) \sigma^{n}\left(a^{2}\right) \sigma^{n-1}(a u)\left[\frac{\sigma\left(a^{2}\right)}{\sigma(a)}\right]^{2 k}\left[\frac{\sigma(a \bar{u})}{\sigma(a u)}\right]^{r-1},
$$

и справедливо равенство

$$
\sum_{r=1}^{n} A(n, r ; x) t^{r-1}=\frac{Z(n ; \mathbf{1},(u, 1, \ldots, 1))}{\sigma^{n^{2}-2 n+1}(a) \sigma^{n}\left(a^{2}\right) \sigma^{n-1}(a u)},
$$


где

$$
x=\left[\frac{\sigma\left(a^{2}\right)}{\sigma(a)}\right]^{2}, \quad t=\frac{\sigma(a \bar{u})}{\sigma(a u)} .
$$

Итак, задача детального перечисления матриц чередующихся знаков сводится к проблеме изучения статистической суммы $Z(n ; \boldsymbol{x}, \boldsymbol{y})$ при $\boldsymbol{x}=\mathbf{1}$ и $\boldsymbol{y}=(u, 1, \ldots, 1)$.

Используя представление для статистической суммы через детерминант ИзергинаКорепина, Куперберг и Цайлбергер были вынуждены преодолевать проблемы, связанные с его сингулярностью при требуемых значениях спектральных параметров. Один из авторов настоящей работы предложил новый метод обращения с детерминантом Изергина-Корепина, справедливый при $a=e^{i \pi / 3}$. Это позволило дать более простое доказательство гипотезы о детальном перечислении матриц чередующихся знаков. В настояшей статье мы используем метод работы [2] для получения формул, описываюших детальные перечисления некоторых классов симметрии матриц чередующихся знаков, и доказываем гипотезу Кутина-Юена.

\section{2. СЛУЧАЙ ПРОИЗВОЛЬНЫХ МАТРИЦ ЧЕРЕДУЮШИХСЯ ЗНАКОВ}

В этом разделе мы описываем метод исследования детальных перечислений, предложенный в работе [2]. Сначала получим новое детерминантное представление для статистической суммы $Z(n ; \boldsymbol{x}, \boldsymbol{y})$, которое справедливо при $a=e^{i \pi / 3}$. В действительности это представление напрямую не связано с задачами перечислений, однако оно позволяет выявить одну полезную симметрию статистической суммы.

Начнем с представления статистической суммы $Z(n ; \boldsymbol{x}, \boldsymbol{y})$ через детерминант Изергина-Корепина. Напомним, что справедливость этого представления базируется на трех следующих леммах.

Лемма 1. Статистическая сумма $Z(n ; \boldsymbol{x}, \boldsymbol{y})$ симметрична по координатам вектора $\boldsymbol{x}$ и по координатам вектора $\boldsymbol{y}$.

ЛЕмма 2. Статистическая сумма $Z(n ; \boldsymbol{x}, \boldsymbol{y})$ удовлетворяет рекуррентному соотношению

$$
\frac{Z\left(n ;\left(x_{1}, \ldots, x_{n-1}, x_{n}\right),\left(y_{1}, \ldots, y_{n-1}, a x_{n}\right)\right)}{Z\left(n-1 ;\left(x_{1}, \ldots, x_{n-1}\right),\left(y_{1}, \ldots, y_{n-1}\right)\right)}=\sigma\left(a^{2}\right) \prod_{k=1}^{n-1} \sigma\left(a \bar{x}_{n} y_{k}\right) \prod_{k=1}^{n-1} \sigma\left(a \bar{x}_{k} y_{n}\right)
$$

ЛЕмма 3. Для каждого $i=1, \ldots, n$ произведение $x_{i}^{n-1} Z(n ; \boldsymbol{x}, \boldsymbol{y})$ является полиномом от $x_{i}^{2}$ степени $n-1$. Для каждого $i=1, \ldots, n$ произведение $y_{i}^{n-1} Z(n ; \boldsymbol{x}, \boldsymbol{y})$ является полином от $y_{i}^{2}$ степени $n-1$.

Ясно, что эти леммы совместно с начальным условием $Z(1 ; \boldsymbol{x}, \boldsymbol{y})=\sigma\left(a^{2}\right)$ определяют по индукции статистические суммы $Z(n ; \boldsymbol{x}, \boldsymbol{y})$ при $n=2,3, \ldots$ Представление $Z(n ; \boldsymbol{x}, \boldsymbol{y})$ через детерминант Изергина-Корепина составляет содержание следуюшей теоремы. 
Теорема 1. Статистическая сумма $Z(n ; \boldsymbol{x}, \boldsymbol{y})$ может бить записана в виде

$$
Z(n ; \boldsymbol{x}, \boldsymbol{y})=\frac{\sigma^{n}\left(a^{2}\right) \prod_{i, j} \alpha\left(x_{i} \bar{y}_{j}\right)}{\prod_{i<j} \sigma\left(\bar{x}_{i} x_{j}\right) \sigma\left(y_{i} \bar{y}_{j}\right)} \operatorname{det} M(n ; \boldsymbol{x}, \boldsymbol{y}),
$$

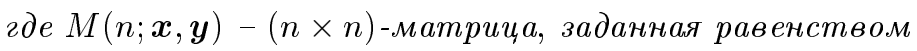

$$
M(n ; \boldsymbol{x}, \boldsymbol{y})_{i j}=\frac{1}{\alpha\left(x_{i} \bar{y}_{j}\right)},
$$

а функиия $\alpha$ определяется следующим образом:

$$
\alpha(x)=\sigma(a x) \sigma(a \bar{x}) .
$$

Эта теорема может быть доказана непосредственной проверкой того, что правая часть равенства (3) удовлетворяет условиям лемм 1-3.

Рассмотрим случай $a=e^{i \pi / 3}$. Заметим, что в этом случае справедливы равенства

$$
1-a+a^{2}=0, \quad 1-\frac{1}{a}+\frac{1}{a^{2}}=0
$$

которые можно использовать для доказательства того, что $\sigma\left(a^{2}\right)=\sigma(a)$ и $\sigma(x) \sigma(a x) \times$ $\sigma\left(a^{2} x\right)=-\sigma\left(x^{3}\right)$. Из последнего равенства следует соотношение

$$
\alpha(x)=-\frac{\sigma\left(x^{3}\right)}{\sigma(x)} .
$$

Используя его и обозначая $u_{2 i-1}=x_{i}, u_{2 i}=y_{i}$, мы переписываем равенство $(3)$ в виде

$$
Z(n ; \boldsymbol{u})=\frac{(-1)^{n} \sigma^{n}(a) \prod_{i, j} \sigma\left(u_{2 i-1}^{3} \bar{u}_{2 j}^{3}\right)}{\prod_{i<j}\left[\sigma\left(\bar{u}_{2 i-1} u_{2 j-1}\right) \sigma\left(u_{2 i} \bar{u}_{2 j}\right)\right] \prod_{i, j} \sigma\left(u_{2 i-1} \bar{u}_{2 j}\right)} \operatorname{det} M(n ; \boldsymbol{u}) .
$$

Введем теперь функцию

$$
F(n ; \boldsymbol{u})=\prod_{\mu<\nu} \sigma\left(u_{\mu} \bar{u}_{\nu}\right) Z(n ; \boldsymbol{u})
$$

Здесь и далее мы считаем, что индексы, обозначаемые греческими буквами, изменяются от 1 до $2 n$. Используя для $Z(n ; \boldsymbol{u})$ детерминантное представление, можно написать

$$
F(n ; \boldsymbol{u})=(-1)^{n} \sigma^{n}(a) \prod_{i, j} \sigma\left(u_{i}^{3} \bar{u}_{i+n}^{3}\right) \operatorname{det} M(n ; \boldsymbol{u}) .
$$

Следующая простая лемма является для нас очень важной.

Лемма 4. Для любого $\mu=1, \ldots, 2$ справедливо равенство

$$
\begin{aligned}
& F\left(n ;\left(u_{1}, \ldots, u_{\mu}, \ldots, u_{2 n}\right)\right)+ \\
& \quad+F\left(n ;\left(u_{1}, \ldots, a^{2} u_{\mu}, \ldots, u_{2 n}\right)\right)+F\left(n ;\left(u_{1}, \ldots, a^{4} u_{\mu}, \ldots, u_{2 n}\right)\right)=0 .
\end{aligned}
$$


ДоКАЗАТЕЛЬСТВО. При $a=e^{i \pi / 3}$ имеем

$$
M(n ; \boldsymbol{x}, \boldsymbol{y})_{i j}=-\frac{\sigma\left(x_{i} \bar{y}_{j}\right)}{\sigma\left(x_{i}^{3} \bar{y}_{j}^{3}\right)}
$$

Используя равенства (4), получаем

$$
\sigma(x)+\sigma\left(a^{2} x\right)+\sigma\left(a^{4} x\right)=0
$$

что дает для первого столбца

$$
M\left(n ;\left(x_{1}, \ldots, x_{n}\right), \boldsymbol{y}\right)_{1 j}+M\left(n ;\left(a^{2} x_{1}, \ldots, x_{n}\right), \boldsymbol{y}\right)_{1 j}+M\left(n ;\left(a^{4} x_{1}, \ldots, x_{n}\right), \boldsymbol{y}\right)_{1 j}=0
$$

Так как $\operatorname{det} M(n ; \boldsymbol{x}, \boldsymbol{y})$ для любого $j$ зависит от $M(n ; \boldsymbol{x}, \boldsymbol{y})_{1 j}$ линейно, то из последнего равенства следует, что

$$
\begin{aligned}
\operatorname{det} M & \left(n ;\left(x_{1}, \ldots, x_{n}\right), \boldsymbol{y}\right)+ \\
\quad & +\operatorname{det} M\left(n ;\left(a^{2} x_{1}, \ldots, x_{n}\right), \boldsymbol{y}\right)+\operatorname{det} M\left(n ;\left(a^{4} x_{1}, \ldots, x_{n}\right), \boldsymbol{y}\right)=0 .
\end{aligned}
$$

Принимая во внимание равенство (7), мы приходим к выводу, что утверждение леммы справедливо для $\mu=1$. Для всех остальных значений $\mu$ применимы эти же аргументы.

Из леммы 3 и соотношения (6) вытекает следуюшая лемма.

Лемма 5. Для любого $\mu=1, \ldots, 2 n$ функция $u_{\mu}^{3 n-2} F(n ; \boldsymbol{u})$ является полиномом степени 3 n- 2 от $u_{\mu}^{2}$.

Следуюшая лемма очевидна.

Лемма 6. Функиия $F(n ; \boldsymbol{u})$ принимает нулевое значение, если $u_{\mu}^{2}=u_{\nu}^{2}$ для некоторых $\mu \neq \nu$.

Покажем теперь, что леммы 4-6 определяют функцию $F(n ; \boldsymbol{u})$ однозначно с точностью до постоянного множителя.

ЛЕмма 7. Функиия $F(n ; \boldsymbol{u})$, удовлетворяющая свойствам, описанным в леммах 4-6, пропорииональна детерминанту матричь

$$
P(n ; \boldsymbol{u})=\left(\begin{array}{ccccc}
u_{1}^{3 n-2} & u_{2}^{3 n-2} & u_{3}^{3 n-2} & \ldots & u_{2 n}^{3 n-2} \\
u_{1}^{3 n-4} & u_{2}^{3 n-4} & u_{3}^{3 n-4} & \ldots & u_{2 n}^{3 n-4} \\
u_{1}^{3 n-8} & u_{2}^{3 n-8} & u_{3}^{3 n-8} & \ldots & u_{2 n}^{3 n-8} \\
\vdots & \vdots & \vdots & \ddots & \vdots \\
u_{1}^{-3 n+2} & u_{2}^{-3 n+2} & u_{3}^{-3 n+2} & \ldots & u_{2 n}^{-3 n+2}
\end{array}\right) .
$$


ДокАЗАТЕльство. Принимая во внимание лемму 5, для фиксированного значения $\mu$ запишем

$$
F(n ; \boldsymbol{u})=\sum_{k=1}^{3 n-1} a_{k}^{(\mu)}\left(u_{1}, \ldots, \hat{u}_{\mu}, \ldots, u_{2 n}\right) u_{\mu}^{3 n-2 k}
$$

знак “^” означает, что соответствующий аргумент пропушен. Из леммы 4 следует, что $a_{k}^{(\mu)}=0$, если $k$ делится на 3 . Поэтому справедливо равенство

$$
F(n ; \boldsymbol{u})=\sum_{\substack{k=1 \\ k \neq 0}}^{3 n-1} a_{k}^{(\mu)}\left(u_{1}, \ldots, \hat{u}_{\mu}, \ldots, u_{2 n}\right) u_{\mu}^{3 n-2 k}
$$

Следовательно, имеется только $2 n$ неизвестных функций $a_{k}^{(\mu)}$. Из леммы 6 следует, что для любого $\nu \neq \mu$ справедливо равенство

$$
\sum_{\substack{k=1 \\ k \neq 0}}^{3 n-1} a_{k}^{(\mu)}\left(u_{1}, \ldots, \hat{u}_{\mu}, \ldots, u_{2 n}\right) u_{\nu}^{3 n-2 k}=0 .
$$

Таким образом, мы имеем систему из $2 n-1$ линейных уравнений для $2 n$ функций $a_{k}^{(\mu)}$. В случае общего положения ранг этой системы равен $2 n-1$. Следовательно, она определяет функции $a_{k}^{(\mu)}$ однозначно с точностью до общего множителя, которьй не может зависеть от $u_{\mu}$. Перебирая все значения $\mu$, мы приходим к выводу, что свойства, описанные в леммах $4-6$, определяют функцию $F(n ; \boldsymbol{u})$ однозначно с точностью до постоянного множителя. Легко убедиться в том, что детерминант матрицы $P(n ; \boldsymbol{u})$ удовлетворяет условиям, описанным в леммах $4-6$. Следовательно, функция $F(n ; \boldsymbol{u})$ должна быть пропорциональна этому детерминанту.

ЛЕмма 8. Детерминант матрищы $P(n ; \boldsymbol{u})$ удовлетворяет рекуррентному соотношению

$$
\frac{\operatorname{det} P\left(n ;\left(u_{1}, \ldots, u_{2 n-2}, u_{2 n-1}, a u_{2 n-1}\right)\right)}{\operatorname{det} P\left(n-1 ;\left(u_{1}, \ldots, u_{2 n-2}\right)\right)}=(-1)^{n} \sigma(a) \prod_{\mu=1}^{2 n-2} \sigma\left(u_{\mu}^{3} \bar{u}_{2 n-1}^{3}\right) .
$$

ДокАЗАТЕЛЬСтво. Вспоминая, что мы рассматриваем случай, когда $a=e^{i \pi / 3}$, можно легко убедиться в том, что детерминант матрицы $P(n ; \boldsymbol{u})$ при $u_{2 n}=a u_{2 n-1}$ совпадает с детерминантом

$$
(-1)^{n+1}\left|\begin{array}{ccccc}
u_{1}^{3 n-2} & u_{2}^{3 n-2} & \ldots & u_{2 n-1}^{3 n-2} & a u_{2 n-1}^{3 n-2} \\
u_{1}^{3 n-4} & u_{2}^{3 n-4} & \ldots & u_{2 n-4}^{3 n-1} & \bar{a} u_{2 n-1}^{3 n-1} \\
u_{1}^{3 n-8} & u_{2}^{3 n-8} & \ldots & u_{2 n-1}^{3 n-8} & a u_{2 n-1}^{3 n-8} \\
\vdots & \vdots & \ddots & \vdots & \vdots \\
u_{1}^{-3 n+8} & u_{2}^{-3 n+8} & \ldots & u_{2 n-1}^{-3 n+8} & \bar{a} u_{2 n-1}^{-3 n+8} \\
u_{1}^{-3 n+4} & u_{2}^{-3 n+4} & \ldots & u_{2 n-1}^{-3 n+4} & a u_{2 n-1}^{-3 n+4} \\
u_{1}^{-3 n+2} & u_{2}^{-3 n+2} & \ldots & u_{2 n-1}^{-3 n+2} & \bar{a} u_{2 n-1}^{-3 n+2}
\end{array}\right|
$$


Вычтем из первой строки этого детерминанта его третью строку, умноженную на $u_{2 n-1}^{6}$, вычтем из второй строки четвертую строку, умноженную опять на $u_{2 n-1}^{6}$, и т.д. пока это возможно. В результате мы получаем следуюшее выражение для $\operatorname{det} P(n ; \boldsymbol{u})$ :

$$
(-1)^{n+1}\left|\begin{array}{ccccc}
\left(u_{1}^{6}-u_{2 n-1}^{6}\right) u_{1}^{3 n-8} & \left(u_{2}^{6}-u_{2 n-1}^{6}\right) u_{2}^{3 n-8} & \ldots & 0 & 0 \\
\left(u_{1}^{6}-u_{2 n-1}^{6}\right) u_{1}^{3 n-10} & \left(u_{2}^{6}-u_{2 n-1}^{6}\right) u_{2}^{3 n-10} & \ldots & 0 & 0 \\
\left(u_{1}^{6}-u_{2 n-1}^{6}\right) u_{1}^{3 n-14} & \left(u_{2}^{6}-u_{2 n-1}^{6}\right) u_{2}^{3 n-14} & \ldots & 0 & 0 \\
\vdots & \vdots & \ddots & \vdots & \vdots \\
\left(u_{1}^{6}-u_{2 n-1}^{6}\right) u_{1}^{-3 n+2} & \left(u_{2}^{6}-u_{2 n-1}^{6}\right) u_{2}^{3 n+2} & \ldots & 0 & 0 \\
u_{1}^{-3 n+4} & u_{2}^{-3 n+4} & \ldots & u_{2 n-1}^{-3 n+4} & a u_{2 n-1}^{-3 n+4} \\
u_{1}^{-3 n+2} & u_{2}^{-3 n+2} & \ldots & u_{2 n-1}^{-3 n+2} & \bar{a} u_{2 n-1}^{-3 n+2}
\end{array}\right| .
$$

Теперь утверждение леммы очевидно.

Теорема 2. При а $=e^{i \pi / 3}$ статистическая сумма $Z(n ; \boldsymbol{u})$ имеет следующее детерминантное представление:

$$
Z(n ; \boldsymbol{u})=(-1)^{n(n-1) / 2} \frac{\sigma^{n}(a)}{\prod_{\mu<\nu} \sigma\left(u_{\mu} \bar{u}_{\nu}\right)} \operatorname{det} P(n ; \boldsymbol{u}) .
$$

ДокАЗАтЕльство. Можно показать, что в нашем случае рекуррентное соотношение из леммы 2 принимает вид

$$
\frac{Z\left(n ;\left(u_{1}, \ldots, u_{2 n-2}, u_{2 n-1}, a u_{2 n-1}\right)\right)}{Z\left(n-1 ;\left(u_{1}, \ldots, u_{2 n-2}\right)\right)}=\sigma(a) \prod_{\mu=1}^{2 n-2} \sigma\left(a u_{\mu} \bar{u}_{2 n-1}\right) .
$$

Учитывая этот факт и лемму 8 , легко видеть, что правая часть соотношения (9) удовлетворяет леммам 1-3. Отсюда следует, что поскольку равенство (9) справедливо для $n=1$, оно справедливо для любого $n$.

Из теоремы 2 , в частности, следует, что статистическая сумма $Z(n ; \boldsymbol{u})$ симметрична по координатам вектора $\boldsymbol{u}$. Возврашаясь к векторам $\boldsymbol{x}$ и $\boldsymbol{y}$, можно сформулировать

СлЕДСтвИЕ 1. При а $=e^{i \pi / 3}$ статистическая сумма $Z(n ; \boldsymbol{x}, \boldsymbol{y})$ симметрична по оббединению координат векторов $\boldsymbol{x}$ и $\boldsymbol{y}$.

Вернемся к задачам перечислений. Как мы отмечали в разделе 1 , для решения этих задач мы должны рассмотреть статистическую сумму $Z(n ; \boldsymbol{x}, \boldsymbol{y})$ при $\boldsymbol{x}=\mathbf{1}, \boldsymbol{y}=1$ или при $\boldsymbol{x}=1, \boldsymbol{y}=(u, 1, \ldots, 1)$. К сожалению, $\operatorname{det} M(n, \boldsymbol{u})$ и $\operatorname{det} P(n, \boldsymbol{u})$ становятся сингулярными при таком выборе спектральных параметров. Куперберг [7] предложил находить значения детерминанта Изергина-Корепина на кривой, включаюшей желаемые значения спектральных параметров. Такой же метод использовал Цайлбергер [12] при доказательстве гипотезы о детальном перечислении матриц чередуюшихся знаков. В работе [2] использован другой метод. Для того чтобы использовать этот метод, определим для фиксированного значения $\mu$ функцию

$$
F^{(\mu)}(n ; \boldsymbol{u})=\prod_{\nu \neq \mu} \sigma\left(u_{\mu} \bar{u}_{\nu}\right) Z(n ; \boldsymbol{u})
$$


и специфицируем значения спектральных параметров следующим образом:

$$
f(n ; u)=F^{(\mu)}(n ;(\underbrace{1, \ldots, 1}_{\mu-1} u, \underbrace{1, \ldots, 1}_{2 n-\mu})) .
$$

Так как статистическая сумма $Z(n ; \boldsymbol{u})$ симметрична по координатам $\boldsymbol{u}$, то функция $f(n ; u)$ не зависит от $\mu$. В самом деле, можно написать

$$
f(n ; u)=\sigma^{2 n-1}(u) Z(n ;(u, 1, \ldots, 1)) .
$$

Лемма 9. Функиия $f(n ; u)$ обладает следующими свойствами.

1. Функиия $u^{3 n-2} f(n ; u)$ является полиномом степени $3 n-2$ от $u^{2}$.

2. Функиия $f(n ; u)$ удовлетворяет соотношению

$$
f(n ; \bar{u})=-f(n ; u) .
$$

3. Для функции $f(n ; u)$ справедливо равенство

$$
f(n ; u)+f\left(n ; a^{2} u\right)+f\left(n ; a^{4} u\right)=0 .
$$

4. Полином Лорана $f(n ; u)$ делится на $\sigma^{2 n-1}(u)$.

По-видимому, необходимо обсудить только свойство 2. В действительности оно следует из равенства

$$
Z\left(n ;\left(\bar{u}_{1}, \bar{u}_{2}, \ldots, \bar{u}_{2 n}\right)\right)=Z\left(n ;\left(u_{1}, u_{2}, \ldots, u_{n}\right)\right),
$$

являюшегося следствием симметрий квадратного льда с граничным условием типа доменной стенки, меняюших левую сторону с правой и верх с низом.

Следуюшая важная лемма была доказана одним из авторов настояшей статьи в работе [13].

ЛЕмма 10. Функиия $f(n ; u)$, удовлетворяющая лемме 9 , пропорциональна функuиu

$$
\varphi(n ; u)=\frac{(-1)^{n-1}}{\sigma(a)\left(\begin{array}{c}
2 n-2 \\
n-1
\end{array}\right)} \sum_{k=0}^{n-1}\left(\begin{array}{c}
n-\frac{4}{3} \\
n-1-k
\end{array}\right)\left(\begin{array}{c}
n-\frac{2}{3} \\
k
\end{array}\right) \sigma\left(u^{3 n-2-6 k}\right) .
$$

Функция $\varphi(n ; u)$ нормирована условием

$$
\varphi(n ; a)=1 .
$$

Нормировочное условие (10) можно проверить, воспользовавшись тождеством

$$
\sum_{k=0}^{n-1}\left(\begin{array}{c}
n-\frac{4}{3} \\
n-1-k
\end{array}\right)\left(\begin{array}{c}
n-\frac{2}{3} \\
k
\end{array}\right)=\left(\begin{array}{c}
2 n-2 \\
n-1
\end{array}\right)
$$


Равенство (2) при $a=e^{i \pi / 3}$ и $u=a$ дает

$$
Z(n ;(a, 1, \ldots, 1))=A(n-1) \sigma^{n^{2}}(a),
$$

где мы учли очевидное равенство $A(n, 1)=A(n-1)$. Следовательно, имеет место равенство

$$
f(n ; u)=A(n-1) \sigma^{n^{2}+2 n-1}(a) \varphi(n ; u),
$$

из которого следует, что

$$
Z(n ;(u, 1, \ldots, 1))=A(n-1) \sigma^{n^{2}+2 n-1}(a) \frac{\varphi(n ; u)}{\sigma(u)^{2 n-1}}
$$

Поучительно переписать равенство (2) при $a=e^{i \pi / 3}$, используя функцию $\varphi(n ; u)$ :

$$
\frac{1}{A(n-1)} \sum_{r=1}^{n} A(n, r) t^{r-1}=\frac{\sigma^{3 n-2}(a) \varphi(n ; u)}{\sigma^{n-1}(a u) \sigma^{2 n-1}(u)}
$$

В работе [2] это соотношение использовалось для доказательства гипотезы о детальном перечислении матриц чередующихся знаков и для того, чтобы получить некоторые результаты по двойным детальным перечислениям матриц чередуюшихся знаков. В следуюшем разделе мы покажем, что описанный выше метод эффективно работает и в других случаях.

\section{3. МАТРИЦЫ ЧЕРЕДУЮШИХСЯ ЗНАКОВ С ВОЗВРАТНОЙ ГРАНИЦЕЙ}

Гипотеза Кутина и Юена относится к вертикально симметричным и внедиагонально симметричным матрицами чередующихся знаков. Можно построить модель квадратного льда, связанную с вертикально симметричными матрицами чередующихся знаков. Насколько нам известно, не существует детерминантной формулы для статистической суммы этой модели для произвольных значений спектральных параметров. Однако можно показать, что множество вертикально симметричных матриц чередующихся знаков можно рассматривать как подмножество множества так называемых матриц чередуюшихся знаков с возвратной границей. Мы дадим определение для этих матриц и рассмотрим статистическую сумму соответствуюшей модели квадратного льда.

Напомним [4], что $(2 n \times n)$-матрища чередующихся знаков с возвратной гранищей выглядит в вертикальном направлении как обычная матрица чередуюшихся знаков. В горизонтальном направлении элементы, равные 1 и -1 , чередуются, если идти по нечетной строке слева направо, а затем по следующей четной строке справа налево. Пример приведен на рис. 7. Состояния соответствующей модели квадратного льда строятся в соответствии с шаблоном, приведенным на рис. 8. Здесь в дополнительных бивалентных возвратных вершинах одно ребро ориентировано внутрь, а другое - наружу. Соответствуюшие веса приведены на рис. 9. Отметим, что введен дополнительный параметр $b$. 


$$
\left(\begin{array}{ccc}
0 & 0 & + \\
0 & + & - \\
+ & - & 0 \\
0 & 0 & + \\
0 & + & 0 \\
0 & 0 & 0
\end{array}\right)
$$

Рис. 7. Пример матрицы чередующихся знаков с возвратной границей.

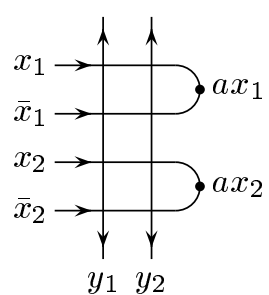

Рис. 8. Квадратный лед с возвратной границей.

$$
\underset{\sigma(b x)}{\rightarrow} x \quad \underset{\sigma(b \bar{x})}{\longrightarrow} x
$$

Рис. 9. Веса возвратных вершин.

Легко показать, что мы имеем взаимно однозначное соответствие состояний и матриц чередующихся знаков с возвратной границей.

Модель квадратного льда с возвратной гранищей впервые изучал Цучия [11]. Он получил детерминантную формулу для статистической суммы. Мы будем использовать модификацию этой формулы, которая была предложена Купербергом [4] и выглядит следующим образом:

$$
Z_{\mathrm{U}}(n ; \boldsymbol{x}, \boldsymbol{y})=\frac{\sigma^{n}\left(a^{2}\right) \prod_{i}\left[\sigma\left(b \bar{y}_{i}\right) \sigma\left(a^{2} x_{i}^{2}\right)\right] \prod_{i, j}\left[\alpha\left(x_{i} \bar{y}_{j}\right) \alpha\left(x_{i} y_{j}\right)\right]}{\prod_{i<j}\left[\sigma\left(\bar{x}_{i} x_{j}\right) \sigma\left(y_{i} \bar{y}_{j}\right)\right] \prod_{i \leqslant j}\left[\sigma\left(\bar{x}_{i} \bar{x}_{j}\right) \sigma\left(y_{i} y_{j}\right)\right]} \operatorname{det} M_{\mathrm{U}}(n ; \boldsymbol{x}, \boldsymbol{y}),
$$

где $(n \times n)$-матрища $M_{\mathrm{U}}$ дается формулой

$$
M_{\mathrm{U}}(n ; \boldsymbol{x}, \boldsymbol{y})_{i, j}=\frac{1}{\alpha\left(x_{i} \bar{y}_{j}\right)}-\frac{1}{\alpha\left(x_{i} y_{j}\right)} .
$$

Обозначим через $A_{\mathrm{U}}(2 n, r ; x, y)$ полный вес матрищ чередующихся знаков с возвратной границей, единственная единица первого столбца которых находится в $r$-й строке, каждый элемент, равный -1 , имеет мультипликативный вес $x$ и каждый разворот, ориентированный вверх, имеет мультипликативный вес $y$. Для того чтобы связать эти 
числа со статистической суммой $Z_{\mathrm{U}}(n ; \boldsymbol{x}, \boldsymbol{y})$, рассмотрим опять случай, когда $\boldsymbol{x}=\mathbf{1}$ и $\boldsymbol{y}=(u, 1, \ldots, 1)$. Пусть для некоторого состояния квадратного льда с возвратной границей соответствуюшая матрица чередуюшихся знаков с возвратной границей имеет единицу на пересечении первого столбца и $r$-й строки. При этом опять в первом столбце имеется одна вершина первого типа, $r-1$ вершина третьего типа и $2 n-r$ вершин шестого типа, где тип опять определяется согласно рис. 6. Следовательно, вклад первого столбца в вес состояния равен $\sigma\left(a^{2}\right) \sigma^{r-1}(a \bar{u}) \sigma^{n-r}(a u)$. Пусть число элементов, равных -1 , в рассматриваемой матрице чередуюшихся знаков равно $k$. В этом случае число единиц в этой матрице чередующихся знаков без первого столбца равно $n-1+k$, а число нулей равно $2 n^{2}-3 n+1-2 k$. Обозначим через $l$ число разворотов, ориентированных вверх. В конце концов можно увидеть, что вес рассматриваемого состояния равен

и справедливо равенство

$$
\sigma^{2 n^{2}-3 n+1}(a) \sigma^{n}\left(a^{2}\right) \sigma^{2 n-1}(a u) \sigma^{n}(a b)\left[\frac{\sigma\left(a^{2}\right)}{\sigma(a)}\right]^{2 k}\left[\frac{\sigma(a \bar{u})}{\sigma(a u)}\right]^{r-1}\left[\frac{\sigma(\bar{a} b)}{\sigma(a b)}\right]^{l},
$$

$$
\sum_{r=1}^{2 n} A_{\mathrm{U}}(2 n, r ; x, y) t^{r-1}=\frac{Z_{\mathrm{U}}(n ; \mathbf{1},(u, 1, \ldots, 1))}{\sigma^{2 n^{2}-3 n+1}(a) \sigma^{n}\left(a^{2}\right) \sigma^{2 n-1}(a u) \sigma^{n}(a b)},
$$

где

$$
x=\left[\frac{\sigma\left(a^{2}\right)}{\sigma(a)}\right]^{2}, \quad y=\frac{\sigma(\bar{a} b)}{\sigma(a b)}, \quad t=\frac{\sigma(a \bar{u})}{\sigma(a u)} .
$$

Удобно ввести модифицированную статистическую сумму

$$
Z_{\mathrm{U}}^{\prime}(n ; \boldsymbol{x}, \boldsymbol{y})=\frac{Z_{\mathrm{U}}(n ; \boldsymbol{x}, \boldsymbol{y})}{\prod_{i}\left[\sigma\left(b \bar{y}_{i}\right) \sigma\left(a^{2} x_{i}^{2}\right)\right]} .
$$

Положим $a=e^{i \pi / 3}$. В этом случае, используя равенство (5), получаем

$$
Z_{\mathrm{U}}^{\prime}(n ; \boldsymbol{u})=(-1)^{n} \frac{\sigma^{n}(a) \prod_{i, j}\left[\sigma\left(u_{2 i-1}^{3} \bar{u}_{2 j}^{3}\right) \sigma\left(u_{2 i-1}^{3} u_{2 j}^{3}\right)\right]}{\prod_{\mu<\nu} \sigma\left(u_{\mu} \bar{u}_{\nu}\right) \prod_{\mu \leqslant \nu} \sigma\left(u_{\mu} u_{\nu}\right)} \operatorname{det} M_{\mathrm{U}}(n ; \boldsymbol{u}),
$$

где мы снова используем обозначения $u_{2 i-1}=x_{i}, u_{2 i}=y_{i}$. Рассмотрим функцию

$$
F_{\mathrm{U}}(n ; \boldsymbol{u})=\prod_{\mu<\nu} \sigma\left(u_{\mu} \bar{u}_{\nu}\right) \prod_{\mu \leqslant \nu} \sigma\left(u_{\mu} u_{\nu}\right) Z_{\mathrm{U}}^{\prime}(n ; \boldsymbol{u})
$$

которая также может быть задана формулой

$$
F_{\mathrm{U}}(n ; \boldsymbol{u})=(-1)^{n} \sigma^{n}(a) \prod_{i, j}\left[\sigma\left(u_{2 i-1}^{3} \bar{u}_{2 j}^{3}\right) \sigma\left(u_{2 i-1}^{3} u_{2 j}^{3}\right)\right] \operatorname{det} M_{\mathrm{U}}(n ; \boldsymbol{u}) .
$$

Имеются следуюшие аналоги лемм 4-6.

ЛЕмма 11. Функиия $F_{\mathrm{U}}(n ; \boldsymbol{u})$ обладает следующими свойствами.

1. Для кажсдого $\mu=1, \ldots, 2$ справедливо равенство

$$
\begin{aligned}
& F_{\mathrm{U}}\left(n ;\left(u_{1}, \ldots, u_{\mu}, \ldots, u_{2 n}\right)\right)+ \\
& \quad+F_{\mathrm{U}}\left(n ;\left(u_{1}, \ldots, a^{2} u_{\mu}, \ldots, u_{2 n}\right)\right)+F_{\mathrm{U}}\left(n ;\left(u_{1}, \ldots, a^{4} u_{\mu}, \ldots, u_{2 n}\right)\right)=0 .
\end{aligned}
$$

2. Для кажсдого $\mu=1, \ldots, 2 n$ функция $u_{\mu}^{6 n-2} F_{\mathrm{U}}(n ; \boldsymbol{u})$ есть полином степени $6 n-2$ om $u_{\mu}^{2}$.

3. Функция $F_{\mathrm{U}}(n ; \boldsymbol{u})$ принимает нулевое значение, если для некоторьх $\mu \neq \nu$ либо $u_{\mu}^{2}=u_{\nu}^{2}$, либо $u_{\mu}^{2}=\bar{u}_{\nu}^{2}$, а такжсе если $u_{\mu}^{4}=1$ длякакого-либо $\mu$. 
ЛЕмма 12. Функиия $F_{\mathrm{U}}(n ; \boldsymbol{u})$, удовлетворяющая свойствам, описанным в лемме 11, пропорииональна детерминанту матриць

$$
P_{\mathrm{U}}(n ; \boldsymbol{u})=\left(\begin{array}{ccccc}
\sigma\left(u_{1}^{6 n-2}\right) & \sigma\left(u_{2}^{6 n-2}\right) & \sigma\left(u_{3}^{6 n-2}\right) & \ldots & \sigma\left(u_{2 n}^{6 n-2}\right) \\
\sigma\left(u_{1}^{6 n-4}\right) & \sigma\left(u_{2}^{6 n-4}\right) & \sigma\left(u_{3}^{6 n-4}\right) & \ldots & \sigma\left(u_{2 n}^{6 n-4}\right) \\
\sigma\left(u_{1}^{8}\right) & \sigma\left(u_{2}^{6 n-8}\right) & \sigma\left(u_{3}^{6 n-8}\right) & \ldots & \sigma\left(u_{2 n}^{6 n-8}\right) \\
\vdots & \vdots & \vdots & \ddots & \vdots \\
\sigma\left(u_{1}^{2}\right) & \sigma\left(u_{2}^{2}\right) & \sigma\left(u_{3}^{2}\right) & \ldots & \sigma\left(u_{2 n}^{2}\right)
\end{array}\right)
$$

ДокАЗАТЕЛЬСтво. Принимая во внимание утверждение 2 леммы 11, для фиксированного значения $\mu$ запишем

$$
F_{\mathrm{U}}(n ; \boldsymbol{u})=\sum_{k=1}^{6 n-1} a_{k}^{(\mu)}\left(u_{1}, \ldots, \hat{u}_{\mu}, \ldots, u_{2 n}\right) u_{\mu}^{6 n-2 k}
$$

Из утверждения 1 леммы 11 следует, что $a_{k}^{(\mu)}=0$, если $k$ делится на 3 . Поэтому имеем равенство

$$
F_{\mathrm{U}}(n ; \boldsymbol{u})=\sum_{\substack{k=1 \\ k \neq 0}}^{6 n-1} a_{k}^{(\mu)}\left(u_{1}, \ldots, \hat{u}_{\mu}, \ldots, u_{2 n}\right) u_{\mu}^{6 n-2 k}
$$

Следовательно, имеется только $4 n$ неизвестных функции $a_{k}^{(\mu)}$. Используя утверждение 3 леммы 11 , для любых $\nu \neq \mu$ получаем, что

$$
\begin{gathered}
\sum_{\substack{k=1 \\
k \neq 0}}^{6 n-1} a_{k}^{(\mu)}\left(u_{1}, \ldots, \hat{u}_{\mu}, \ldots, u_{2 n}\right) u_{\nu}^{6 n-2 k}=0 \\
\sum_{\substack{k=1 \\
k \neq 0}}^{6 n-1} a_{k}^{(\mu)}\left(u_{1}, \ldots, \hat{u}_{\mu}, \ldots, u_{2 n}\right) \bar{u}_{\nu}^{6 n-2 k}=0 \\
\sum_{\bmod 3)}
\end{gathered}
$$

и, кроме того,

$$
\sum_{\substack{k=1 \\ k \neq 0(\bmod 3)}}^{6 n-1} a_{k}^{(\mu)}\left(u_{1}, \ldots, \hat{u}_{\mu}, \ldots, u_{2 n}\right)=0 .
$$

Таким образом, мы имеем систему из $4 n-1$ линейных уравнений для $4 n$ функций $a_{k}^{(\mu)}$. В случае обшего положения ранг этой системы равен $4 n-1$. Следовательно, она определяет функции $a_{k}^{(\mu)}$ однозначно с точностью до обшего множителя, которьй не может зависеть от $u_{\mu}$. Перебирая все значения $\mu$, мы приходим к заключению, что условия, описанные в лемме 11 , определяют функцию $F_{\mathrm{U}}(n ; \boldsymbol{u})$ однозначно с точностью до постоянного множителя. Нетрудно убедиться в том, что детерминант матрицы $P_{\mathrm{U}}(n ; \boldsymbol{u})$ удовлетворяет условиям, описанным в лемме 11. Следовательно, функция $F_{\mathrm{U}}(n ; \boldsymbol{u})$ должна быть пропорциональной этому детерминанту. 
Лемма 13. Детерминант матрицы $P_{\mathrm{U}}(n ; \boldsymbol{u})$ удовлетворяет рекуррентному соотношению

$$
\begin{aligned}
& \frac{\operatorname{det} P_{\mathrm{U}}\left(n ;\left(u_{1}, \ldots, u_{2 n-2}, u_{2 n-1}, a u_{2 n-1}\right)\right)}{\operatorname{det} P_{\mathrm{U}}\left(n-1 ;\left(u_{1}, \ldots, u_{2 n-2}\right)\right)}= \\
& \quad=\sigma(a) \sigma\left(u_{2 n-1}^{6}\right) \prod_{\mu=1}^{2 n-2}\left[\sigma\left(u_{\mu} \bar{u}_{2 n-1}\right) \sigma\left(u_{\mu} u_{2 n-1}\right)\right] .
\end{aligned}
$$

ДокАЗАТЕльство. Рассмотрим матрищу

Можно убедиться в том, что

$$
P_{\mathrm{U}}^{\prime}(n ; \boldsymbol{u})=\left(\begin{array}{cccc}
\sigma\left(u_{1}^{6 n-2}\right) & \ldots & \sigma\left(u_{2 n-1}^{6 n-2}\right) & \sigma\left(u_{2 n-2}^{6 n-2}\right) \\
\sigma\left(u_{1}^{6 n-8}\right) & \ldots & \sigma\left(u_{2 n-1}^{6 n-8}\right) & \sigma\left(u_{2 n}^{6 n-8}\right) \\
\sigma\left(u_{1}^{6 n-14}\right) & \ldots & \sigma\left(u_{2 n-1}^{6 n-14}\right) & \sigma\left(u_{2 n}^{6 n-14}\right) \\
\vdots & \ddots & \vdots & \vdots \\
\sigma\left(u_{1}^{-6 n+10}\right) & \ldots & \sigma\left(u_{2 n-1}^{-6 n+10}\right) & \sigma\left(u_{2 n}^{-6 n+10}\right) \\
\sigma\left(u_{1}^{-6 n+4}\right) & \ldots & \sigma\left(u_{2 n-1}^{-6 n+4}\right) & \sigma\left(u_{2 n}^{-6 n+4}\right)
\end{array}\right) .
$$

$\operatorname{det} P_{\mathrm{U}}(n ; \boldsymbol{u})=(-1)^{n} \operatorname{det} P_{\mathrm{U}}^{\prime}(n ; \boldsymbol{u})$.

При $a=e^{i \pi / 3}$

$$
\operatorname{det} P_{\mathrm{U}}^{\prime}(n ; \boldsymbol{u})=\left|\begin{array}{cccc}
\sigma\left(u_{1}^{6 n-2}\right) & \ldots & \sigma\left(u_{2 n-1}^{6 n-2}\right) & \sigma\left(\bar{a}^{2} u_{2 n-1}^{6 n-2}\right) \\
\sigma\left(u_{1}^{6 n-8}\right) & \ldots & \sigma\left(u_{2 n-1}^{6 n-8}\right) & \sigma\left(\bar{a}^{2} u_{2 n-1}^{6 n-8}\right) \\
\vdots & \ddots & \vdots & \vdots \\
\sigma\left(u_{1}^{-6 n+16}\right) & \ldots & \sigma\left(u_{2 n-1}^{-6 n+16}\right) & \sigma\left(\bar{a}^{2} u_{2 n-1}^{-6 n+16}\right) \\
\sigma\left(u_{1}^{-6 n+10}\right) & \ldots & \sigma\left(u_{2 n-1}^{-6 n+10}\right) & \sigma\left(\bar{a}^{2} u_{2 n-1}^{-6 n+10}\right) \\
\sigma\left(u_{1}^{-6 n+4}\right) & \ldots & \sigma\left(u_{2 n-1}^{-6 n+4}\right) & \sigma\left(\bar{a}^{2} u_{2 n-1}^{-6 n+4}\right)
\end{array}\right|
$$

Вычтем вторую строку, умноженную на $u_{2 n-1}^{6}+u_{2 n-1}^{-6}$, из первой строки и добавим затем третью строку. Повторим такую процедуру, начиная со второй строки, и т.д., пока это возможно. В результате получаем детерминант

$$
\left|\begin{array}{cccc}
\sigma\left(u_{1}^{3} \bar{u}_{2 n-1}^{3}\right) \sigma\left(u_{1}^{3} u_{2 n-1}^{3}\right) \sigma\left(u_{1}^{6 n-8}\right) & \ldots & 0 & 0 \\
\sigma\left(u_{1}^{3} \bar{u}_{2 n-1}^{3}\right) \sigma\left(u_{1}^{3} u_{2 n-1}^{3}\right) \sigma\left(u_{1}^{6 n-14}\right) & \ldots & 0 & 0 \\
\vdots & \ddots & \vdots & \vdots \\
\sigma\left(u_{1}^{3} \bar{u}_{2 n-1}^{3}\right) \sigma\left(u_{1}^{3} u_{2 n-1}^{3}\right) \sigma\left(u_{1}^{-6 n+10}\right) & \ldots & 0 & 0 \\
\sigma\left(u_{1}^{-6 n+10}\right) & \ldots & \sigma\left(u_{2 n-1}^{-6 n+10}\right) & \sigma\left(\bar{a}^{2} u_{2 n-1}^{-6 n+10}\right) \\
\sigma\left(u_{1}^{-6 n+4}\right) & \ldots & \sigma\left(u_{2 n-1}^{-6 n+4}\right) & \sigma\left(\bar{a}^{2} u_{2 n-1}^{-6 n+4}\right)
\end{array}\right| .
$$

Последнее выражение приводит к равенству

$$
\begin{aligned}
& \frac{\operatorname{det} P_{\mathrm{U}}^{\prime}\left(n ;\left(u_{1}, \ldots, u_{2 n-2}, u_{2 n-1}, a u_{2 n}\right)\right)}{\operatorname{det} P_{\mathrm{U}}^{\prime}\left(n-1 ;\left(u_{1}, \ldots, u_{2 n-2}\right)\right)}= \\
& \quad=-\sigma(a) \sigma\left(u_{2 n-1}^{6}\right) \prod_{\mu=1}^{2 n-2}\left[\sigma\left(u_{\mu} \bar{u}_{2 n-1}\right) \sigma\left(u_{\mu} u_{2 n-1}\right)\right]
\end{aligned}
$$

из которого следует утверждение леммы. 
ТЕОремА 3. При $а=e^{i \pi / 3}$ функиия $Z_{\mathrm{U}}^{\prime}(n ; \boldsymbol{u})$ имеет следующее детерминантное представление:

$$
Z_{\mathrm{U}}^{\prime}(n ; \boldsymbol{u})=\frac{\sigma^{n}(a)}{\prod_{\mu<\nu} \sigma\left(u_{\mu} \bar{u}_{\nu}\right) \prod_{\mu \leqslant \nu} \sigma\left(u_{\mu} u_{\nu}\right)} \operatorname{det} P_{\mathrm{U}}(n ; \boldsymbol{u}) .
$$

Вышеприведенную теорему можно доказать, используя лемму 13 и соответствуюшее рекуррентное соотношение для $Z_{\mathrm{U}}(n ; \boldsymbol{x}, \boldsymbol{y})$, найденное Купербергом [4].

СЛЕДСТВИЕ 2. При $а=e^{i \pi / 3}$ полином Лорана

$$
\frac{Z_{\mathrm{U}}(n ; \boldsymbol{x}, \boldsymbol{y})}{\prod_{i=1}^{n} \sigma\left(y_{i} \bar{b}\right) \sigma\left(x_{i}^{2} a^{2}\right)}
$$

симметричен по обвединению координат векторов $\boldsymbol{x}$ и $\boldsymbol{y}$

Введем функцию $f_{\mathrm{U}}(n ; u)=\sigma^{4 n-2}(u) \sigma\left(u^{2}\right) Z_{\mathrm{U}}^{\prime}(n ;(u, 1, \ldots, 1))$.

ЛЕмма 14. Функиия $f_{\mathrm{U}}(n ; u)$ обладает следующими свойствами.

1. Функиия $u^{6 n-2} f_{\mathrm{U}}(n ; u)$ является полиномом степени $6 n-2$ от $u^{2}$.

2. Функиия $f_{\mathrm{U}}(n ; u)$ удовлетворяет соотношению

$$
f(n ; \bar{u})=-f(n ; u) .
$$

3. Для функции $f_{\mathrm{U}}(n ; u)$ справедливо равенство

$$
f_{\mathrm{U}}(n ; u)+f_{\mathrm{U}}\left(n ; a^{2} u\right)+f_{\mathrm{U}}\left(n ; a^{4} u\right)=0 .
$$

4. Полином Лорана $f_{\mathrm{U}}(n ; u)$ делится на $\sigma^{4 n-2}(u)$ ина $\sigma\left(u^{2}\right)$.

Свойство 2 следует в этом случае, например, из детерминантного представления для функции $Z_{\mathrm{U}}^{\prime}(n ; \boldsymbol{u})$, даваемого теоремой 3 . Все остальные свойства очевидны.

Так как $\sigma\left(u^{2}\right)=(u+\bar{u}) \sigma(u)$, то на самом деле функция $f_{\mathrm{U}}(n ; u)$ делится на $\sigma^{4 n-1}(u)$. Сравнивая теперь леммы 14 и 9, а также учитывая лемму 10, приходим к заключению, что функция $f_{\mathrm{U}}(n ; u)$ пропорциональна функции $\varphi(2 n ; u)$. Найдем соответствующий коэффициент пропорциональности. Для этого положим $a=e^{i \pi / 3}$ в равенстве (12). Принимая во внимание очевидное свойство

$$
A_{\mathrm{U}}(2 n, 1 ; 1, y)=y A_{\mathrm{U}}(2 n-2 ; 1, y),
$$

получаем соотношение

$$
A_{\mathrm{U}}(2 n-2 ; 1, y)=\frac{Z_{\mathrm{U}}(n ; \mathbf{1},(a, 1, \ldots, 1))}{\sigma^{2 n^{2}}(a) \sigma^{n-1}(a b) \sigma(\bar{a} b)},
$$

которое после некоторых простых преобразований дает

$$
f_{\mathrm{U}}(n ; u)=A_{\mathrm{U}}(2 n-2 ; 1, y) \frac{\sigma^{2 n^{2}+3 n-1}(a) \sigma^{n-1}(a b)}{\sigma^{n-1}(b)} \varphi(2 n ; u) .
$$

Используя это соотношение, нетрудно показать, что равенство (12) при $a=e^{i \pi / 3}$ может быть записано в виде

$$
\frac{1}{A_{\mathrm{U}}(2 n-2 ; 1, y)} \sum_{r=1}^{2 n} A_{\mathrm{U}}(2 n, r ; 1, y) t^{r-1}=\frac{\sigma(b \bar{u}) \sigma^{6 n-2}(a) \varphi(2 n ; u)}{\sigma(b a) \sigma^{2 n-1}(a u) \sigma^{4 n-2}(u) \sigma\left(u^{2}\right)} .
$$

Сравнивая (14) и (11), мы приходим к следуюшей теореме. 
Теорема 4. Справедливо следующее равенство:

$$
\begin{aligned}
& \frac{1}{A_{\mathrm{U}}(2 n-2 ; 1, y)} \sum_{r=1}^{2 n} A_{\mathrm{U}}(2 n, r ; 1, y) t^{r-1}=\frac{1}{A(2 n-1)} \frac{t+y}{t+1} \sum_{r=1}^{2 n} A(2 n, r) t^{r-1} \text {. } \\
& \underset{r}{\rightarrow \uparrow} \rightarrow \stackrel{\uparrow}{\rightarrow \uparrow}
\end{aligned}
$$

Рис. 10. Состояния квадратного льда с возвратной границей для $n=1$.

Утверждение теоремы 4 при $t=1$ принимает вид

$$
\frac{A_{\mathrm{U}}(2 n ; 1, y)}{A_{\mathrm{U}}(2 n-2 ; 1, y)}=\frac{1}{2}(1+y) \frac{A(2 n)}{A(2 n-1)} .
$$

Для $n=1$ имеются два состояния квадратного льда с возвратной гранищей: одно состояние имеет разворот, ориентированный вверх, а другое - разворот, ориентированный вниз (см. рис. 10). Поэтому справедливо соотношение $A_{\mathrm{U}}(2 ; 1, y)=(1+y)$, и равенство (16) дает

$$
A_{\mathrm{U}}(2 n ; 1, y)=\frac{1}{2^{n}}(1+y)^{n} \prod_{k=1}^{n} \frac{A(2 k)}{A(2 k-1)} .
$$

Используя знаменитое соотношение

$$
\frac{A(n)}{A(n-1)}=\frac{(3 n-2) !(n-1) !}{(2 n-1) !(2 n-2) !}
$$

можно переписать предыдушую формулу для $A_{\mathrm{U}}(2 n ; 1, y)$ в виде

$$
A_{\mathrm{U}}(2 n ; 1, y)=\frac{1}{2^{n}}(1+y)^{n} \prod_{k=1}^{n} \frac{(6 k-2) !(2 k-1) !}{(4 k-1) !(4 k-2) !} .
$$

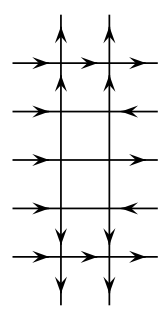

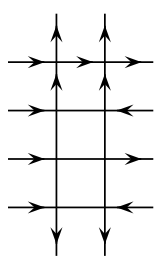

б

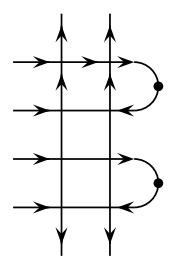

B

Рис. 11. Преобразование состояния квадратного льда, соответствующего вертикально симметричной матрице чередующихся знаков, в состояние квадратного льда с возвратной границей. 
Обсудим теперь связь матриц чередующихся знаков с возвратной границей и вертикально симметричных матриц чередующихся знаков. Нетрудно увидеть, что центральньй столбец вертикально симметричной $((2 n+1) \times(2 n+1))$-матрицы чередующихся знаков состоит из чередующихся элементов, равных 1 и -1 , и он один и тот же для всех таких матриц. Следовательно, вертикально симметричная матрица чередующихся знаков однозначно определяется своей самой левой $((2 n+1) \times n)$-подматрицей. Поэтому с вертикально симметричной матрицей чередующихся знаков можно связать область квадратного льда размером $(2 n+1) \times n$ со следующими граничными условиями. С левой стороны ребра ориентированы внутрь, снизу и сверху - наружу, а с правой стороны ориентация ребер чередуется (см. рис. 11a). Заметим также, что вид верхней и нижней строк у вертикально симметричной матрицы чередуюшихся знаков фиксирован. Поэтому также фиксированны и ориентации ребер, принадлежаших соответствуюшим вершинам. Этот факт отражен на рис. 11. В действительности верхнюю и нижнюю строки можно не рассматривать вообще. Однако принято их сохранять, и мы будем поступать так же.

Любое состояние квадратного льда, соответствуюшее вертикально симметричной матрице чередующихся знаков, можно преобразовать в состояние квадратного льда с возвратной гранищей посредством двух следуюших шагов. Сначала удалим самьй нижний ряд вершин (см. рис. 11б). Затем соединим попарно чередуюшиеся ребра с правой стороны (см. рис. 11в). Заметим, что в полученном состоянии все развороты ориентированы вниз. Ясно, что любое состояние квадратного льда с возвратной границей, все развороты которого ориентированы вниз, может быть преобразовано в состояние квадратного льда, соответствуюшее вертикально симметричной матрице чередующихся знаков. Таким образом, множество вертикально симметричных матриц чередующихся знаков может быть отождествлено с подмножеством матриц чередующихся знаков с возвратной гранищей.

Возврашаясь к задачам перечислений, запишем равенство

$$
A_{\mathrm{V}}(2 n+1, r)=A_{\mathrm{U}}(2 n, r ; 1,0)
$$

которое прямо вытекает из соответствия между вертикально симметричными матрицами чередующихся знаков и матрицами чередующихся знаков с возвратной границей, обсужденного только что. Имея в виду это равенство, мы видим, что из соотношения (17) следует, что

$$
A_{\mathrm{V}}(2 n+1)=\frac{1}{2^{n}} \prod_{k=1}^{n} \frac{(6 k-2) !(2 k-1) !}{(4 k-1) !(4 k-2) !}
$$

и можно написать

$$
A_{\mathrm{U}}(2 n ; 1, y)=(1+y)^{n} A_{\mathrm{V}}(2 n+1) .
$$

Это равенство является частным случаем равенства

$$
A_{\mathrm{U}}(2 n ; x, y)=(1+y)^{n} A_{\mathrm{V}}(2 n+1 ; x),
$$


доказанного Купербергом [4]. Формула (19) эквивалентна рекуррентному соотношению

$$
\frac{A_{\mathrm{V}}(2 n+1)}{A_{\mathrm{V}}(2 n-1)}=\frac{\left(\begin{array}{c}
6 n-2 \\
2 n
\end{array}\right)}{2\left(\begin{array}{c}
4 n-1 \\
2 n
\end{array}\right)}
$$

предугаданному Робинсом [14]. Можно показать, что соотношение (19) эквивалентно формуле для $A_{\mathrm{V}}(2 n+1)$, полученной Купербергом [4].

Рассмотрим теперь утверждение теоремы 4 для случая произвольного $t$. Перепишем (15) в виде

$$
A(2 n-1)(t+1) \sum_{r=1}^{2 n} A_{\mathrm{U}}(2 n, r ; 1, y) t^{r-1}=A_{\mathrm{U}}(2 n-2 ; 1, y)(t+y) \sum_{r=1}^{2 n} A(2 n, r) t^{r-1}
$$

и приравняем коэффициенты, стояшие при различных степенях $t$. В результате получаем следующие равенства:

$$
\begin{aligned}
& A(2 n-1)\left[A_{\mathrm{U}}(2 n, r-1 ; 1, y)+A_{\mathrm{U}}(2 n, r ; 1, y)\right]= \\
& \quad=A_{\mathrm{U}}(2 n-2 ; 1, y)[A(2 n, r-1)+y A(2 n, r)], \quad r=2,3, \ldots, 2 n .
\end{aligned}
$$

Используя соотношения (13) и (20), получаем следуюшее решение этого рекуррентного соотношения:

$$
A_{\mathrm{U}}(2 n, r ; 1, y)=\frac{(1+y)^{n-1} A_{\mathrm{V}}(2 n-1)}{A(2 n-1)}\left[y A(2 n, r)+\sum_{k=1}^{r-1}(-1)^{r+k-1}(1-y) A(2 n, k)\right] .
$$

Для $y=1$ имеем

$$
\begin{aligned}
& A_{\mathrm{U}}(2 n, r)=2^{n-1} \frac{A_{\mathrm{V}}(2 n-1)}{A(2 n-1)} A(2 n, r) .
\end{aligned}
$$

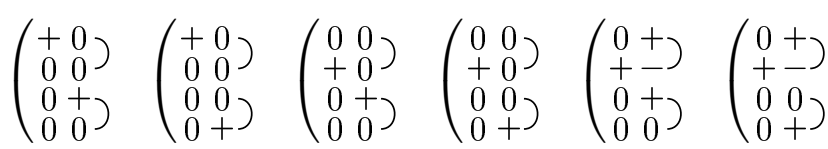

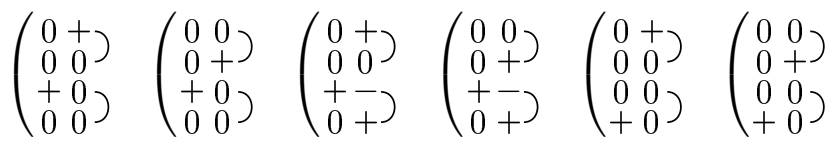

Рис. 12. Все матрицы чередующихся знаков с возвратной границей для $n=2$. 
Рассмотрим для примера случай $n=2$. В этом случае имеется 12 матриц чередуюшихся знаков с возвратной гранищей (рис. 12). Числа $A_{\mathrm{U}}(2 n, r)$ суть $2,4,4,2$. Соответствующие числа $A(2 n, r)$ суть $7,14,14,7$. Легко найти, что $A_{\mathrm{V}}(3)=1$ и $A(3)=7$. Следовательно, наше соотношение в этом случае справедливо.

Соотношение (22) при $y=0$ становится рекуррентным соотношением

$$
A(2 n-1)\left[A_{\mathrm{V}}(2 n+1, r-1)+A_{\mathrm{V}}(2 n+1, r)\right]=A_{\mathrm{V}}(2 n-1) A(2 n, r-1),
$$

которое имеет следующее решение:

$$
A_{\mathrm{V}}(2 n+1, r)=\frac{A_{\mathrm{V}}(2 n-1)}{A(2 n-1)} \sum_{k=1}^{r-1}(-1)^{r+k-1} A(2 n, k) .
$$

Используя формулу [12], [2]

$$
\frac{A(n, r)}{A(n-1)}=\frac{1}{(2 n-2) !} \frac{(n+r-2) !(2 n-r-1) !}{(r-1) !(n-r) !}
$$

приходим к равенству

$$
A_{\mathrm{V}}(2 n+1, r)=\frac{A_{\mathrm{V}}(2 n-1)}{(4 n-2) !} \sum_{k=1}^{r-1}(-1)^{r+k-1} \frac{(2 n+k-2) !(4 n-k-1) !}{(k-1) !(2 n-k) !}
$$

Отметим, что для производящих функций справедливо равенство

$$
\frac{1}{A_{\mathrm{V}}(2 n-1)} \sum_{r=1}^{2 n} A_{\mathrm{V}}(2 n+1, r) t^{r-1}=\frac{1}{A(2 n-1)} \frac{t}{t+1} \sum_{r=1}^{2 n} A(2 n, r) t^{r-1}
$$

которое сразу следует из утверждения теоремы 4 , если положить $y=0$ и учесть равенство (18).

Сформулируем главные результаты по перечислениям, полученные в этом разделе, как следствие теоремы 4.

СледСтвИЕ 3. Справедливы следующие равенства:

$$
\begin{aligned}
A_{\mathrm{V}}(2 n+1) & =\frac{1}{2^{n}} \prod_{k=1}^{n} \frac{(6 k-2) !(2 k-1) !}{(4 k-1) !(4 k-2) !}, \\
A_{\mathrm{U}}(2 n) & =2^{n} A_{\mathrm{V}}(2 n+1), \\
A_{\mathrm{U}}(2 n, r) & =2^{n-1} \frac{A_{\mathrm{V}}(2 n-1)}{A(2 n-1)} A(2 n, r), \\
A_{\mathrm{V}}(2 n+1, r) & =\frac{A_{\mathrm{V}}(2 n-1)}{(4 n-2) !} \sum_{k=1}^{r-1}(-1)^{r+k-1} \frac{(2 n+k-2) !(4 n-k-1) !}{(k-1) !(2 n-k) !} .
\end{aligned}
$$




\section{4. ВНЕДИАГОНАЛЬНО СИММЕТРИЧНЫЕ МАТРИЦЫ ЧЕРЕДУЮЩИХСЯ ЗНАКОВ}

Напомним, что внедиагонально симметричная матрица чередуюшихся знаков - это матрица чередуюшихся знаков, которая совпадает с транспонированной матрицей и имеет нулевую диагональ. Пример соответветствуюшего шаблона для модели квадратного льда приведен на рис. 13. Эта модель была предложена Купербергом [4], нашедшим также следуюшее представление для статистической суммы через пфафффиан:

$$
Z_{\mathrm{O}}(n ; \boldsymbol{u})=\frac{\sigma^{n}\left(a^{2}\right) \prod_{\mu<\nu} \alpha\left(u_{\mu} u_{\nu}\right)}{\prod_{\mu<\nu} \sigma\left(\bar{u}_{\mu} u_{\nu}\right)} \operatorname{Pf} M_{\mathrm{O}}(n ; \boldsymbol{u}),
$$

где $M_{\mathrm{O}}(n ; \boldsymbol{x})-(2 n \times 2 n)$-матрица, матричные элементы которой даются равенством

$$
M_{\mathrm{O}}(n ; \boldsymbol{u})_{\mu \nu}=\frac{\sigma\left(\bar{u}_{\mu} u_{\nu}\right)}{\alpha\left(u_{\mu} u_{\nu}\right)}
$$

Нетрудно получить соотношение

$$
\sum_{r=2}^{2 n} A_{\mathrm{O}}(2 n, r ; x) t^{r-2}=\frac{Z_{\mathrm{O}}(n ;(\bar{u}, 1, \ldots, 1))}{\sigma^{2 n^{2}-2 n+1}(a) \sigma^{n}\left(a^{2}\right) \sigma^{2 n-2}(a u)},
$$

где

$$
x=\left[\frac{\sigma\left(a^{2}\right)}{\sigma(a)}\right]^{2}, \quad t=\frac{\sigma(a \bar{u})}{\sigma(a u)} .
$$

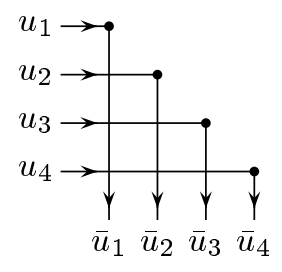

Рис. 13. Квадратный лед с внедиагонально симметричной границей.

Предположим опять, что $a=e^{i \pi / 3}$. В этом случае, используя равенство (5), перепишем статистическую сумму в виде

$$
Z_{\mathrm{O}}(n ; \boldsymbol{u})=(-1)^{n} \frac{\sigma^{n}\left(a^{2}\right) \prod_{\mu<\nu} \sigma\left(u_{\mu}^{3} u_{\nu}^{3}\right) \prod_{\mu} \sigma\left(u_{\mu}^{2}\right)}{\prod_{\mu<\nu} \sigma\left(\bar{u}_{\mu} u_{\nu}\right) \prod_{\mu \leqslant \nu} \sigma\left(u_{\mu} u_{\nu}\right)} \operatorname{Pf} M_{\mathrm{O}}(n ; \boldsymbol{u}) .
$$

Определим функцию

$$
F_{\mathrm{O}}(n ; \boldsymbol{u})=\sigma(a)^{-n} \prod_{\mu<\nu} \sigma\left(\bar{u}_{\mu} u_{\nu}\right) \prod_{\mu \leqslant \nu} \sigma\left(u_{\mu} u_{\nu}\right) Z_{\mathrm{O}}(n ; \boldsymbol{u}),
$$

которая также может быть задана следующим образом:

$$
F_{\mathrm{O}}(n ; \boldsymbol{u})=(-1)^{n} \prod_{\mu<\nu} \sigma\left(u_{\mu}^{3} u_{\nu}^{3}\right) \prod_{\mu} \sigma\left(u_{\mu}^{2}\right) \operatorname{Pf} M_{\mathrm{O}}(n ; \boldsymbol{u}) .
$$


ЛЕмма 15. Функиия $F_{\mathrm{O}}(n ; \boldsymbol{u})$ обладает следующими свойствами.

1. Для любого $\mu=1, \ldots, 2$ справедливо равенство

$$
\begin{aligned}
& F_{\mathrm{O}}\left(n ;\left(u_{1}, \ldots, u_{\mu}, \ldots, u_{2 n}\right)\right)+ \\
& \quad+F_{\mathrm{O}}\left(n ;\left(u_{1}, \ldots, a^{2} u_{\mu}, \ldots, u_{2 n}\right)\right)+F_{\mathrm{O}}\left(n ;\left(u_{1}, \ldots, a^{4} u_{\mu}, \ldots, u_{2 n}\right)\right)=0
\end{aligned}
$$

2. Для любого $\mu=1, \ldots, 2 n$ функиия $u_{\mu}^{6 n-2} F_{\mathrm{O}}(n ; \boldsymbol{u})$ является полиномом степени 6 п -2 от $u_{\mu}^{2}$.

3. Функция $F_{\mathrm{O}}(n ; \boldsymbol{u})$ принимает нулевое значение, если для некоторых $\mu \neq \nu$ либо $u_{\mu}^{2}=u_{\nu}^{2}$, либо $u_{\mu}^{2}=\bar{u}_{\nu}^{2}$, а также если $u_{\mu}^{4}=1$ длякакого-либо $\mu$.

ДокАЗАтЕльство. Для доказательства утверждения 1 заметим, что при $a=e^{i \pi / 3}$ справедливо равенство

$$
M_{\mathrm{O}}(n ; \boldsymbol{u})_{\mu \nu}=-\frac{\sigma\left(\bar{u}_{\mu} u_{\nu}\right) \sigma\left(u_{\mu} u_{\nu}\right)}{\sigma\left(u_{\mu}^{3} u_{\nu}^{3}\right)} .
$$

Можно убедиться в том, что

$$
\sigma\left(u_{\mu}^{2}\right) M_{\mathrm{O}}(n ; \boldsymbol{u})_{\mu \nu}=-\frac{\sigma\left(u_{\mu}^{2}\right) \sigma\left(u_{\nu}^{2}\right)-\sigma\left(u_{\mu}^{4}\right)}{\sigma\left(u_{\mu}^{3} u_{\nu}^{3}\right)} .
$$

Принимая во внимание равенство $\sigma(x)+\sigma\left(a^{8} x\right)+\sigma\left(a^{16} x\right)=0$, которое следует из (8), получаем

$$
\begin{aligned}
\sigma\left(u_{1}^{2}\right) & M_{\mathrm{O}}\left(n ;\left(u_{1}, \ldots, u_{2 n}\right)\right)_{1 \nu}+ \\
& +\sigma\left(a^{4} u_{1}^{2}\right) M_{\mathrm{O}}\left(n ;\left(a^{2} u_{1}, \ldots, u_{2 n}\right)\right)_{1 \nu}+\sigma\left(a^{8} u_{1}^{2}\right) M_{\mathrm{O}}\left(n ;\left(a^{4} u_{1}, \ldots, u_{2 n}\right)\right)_{1 \nu}=0
\end{aligned}
$$

Так как $\operatorname{Pf} M_{\mathrm{O}}(n ; \boldsymbol{u})$ для каждого $\nu$ зависит от $M_{\mathrm{O}}(n ; \boldsymbol{u})_{1 \nu}$ линейно, то из последнего равенства следует, что

$$
\begin{aligned}
\sigma\left(u_{1}^{2}\right) & \operatorname{Pf} M_{\mathrm{O}}\left(n ;\left(u_{1}, \ldots, u_{2 n}\right)\right)+ \\
& +\sigma\left(a^{4} u_{1}^{2}\right) \operatorname{Pf} M_{\mathrm{O}}\left(n ;\left(a^{2} u_{1}, \ldots, u_{2 n}\right)\right)+\sigma\left(a^{8} u_{1}^{2}\right) \operatorname{Pf} M_{\mathrm{O}}\left(n ;\left(a^{4} u_{1}, \ldots, u_{2 n}\right)\right)=0 .
\end{aligned}
$$

Мы видим, что утверждение 1 леммы справедливо для $\mu=1$. Для всех остальных значений $\mu$ доказательство аналогично. Утверждения 2 и 3 очевидны.

Сравнивая леммы 15 и 11 и принимая во внимание лемму 12, мы приходим к выводу, что функция $F_{\mathrm{O}}(n ; \boldsymbol{u})$ пропорциональна $\operatorname{det} P_{\mathrm{U}}(n ; \boldsymbol{u})$.

ТЕОРема 5. При $а=e^{i \pi / 3}$ функиия $Z_{\mathrm{O}}(n ; \boldsymbol{u})$ имеет следующее детерминантное представление:

$$
Z_{\mathrm{O}}(n ; \boldsymbol{u})=\frac{\sigma^{n}(a)}{\prod_{\mu<\nu} \sigma\left(u_{\mu} \bar{u}_{\nu}\right) \prod_{\mu \leqslant \nu} \sigma\left(u_{\mu} u_{\nu}\right)} \operatorname{det} P_{\mathrm{U}}(n ; \boldsymbol{u}) .
$$

Доказательство теоремы 5 основано на использовании леммы 13 и рекуррентного соотношения для статистической суммы $Z_{\mathrm{O}}(n ; \boldsymbol{u})$, полученного Купербергом [4]. 
СледСТВИЕ 4. Статистическая сумма $Z_{\mathrm{O}}(n ; \boldsymbol{u})$ совпадает с модифицированной статистической суммой $Z_{\mathrm{U}}^{\prime}(n ; \boldsymbol{u})$.

Определим функцию

$$
f_{\mathrm{O}}(n ; u)=\sigma^{4 n-2}(u) \sigma\left(u^{2}\right) Z_{\mathrm{O}}(n ;(u, 1, \ldots, 1))
$$

Функция $f_{\mathrm{O}}(n ; u)$ обладает свойствами функции $f_{\mathrm{U}}(n ; u)$, которые описаны в лемме 14 . Поэтому она пропорциональна функции $\varphi(2 n ; u)$. Как и в случае матриц чередуюшихся знаков с возвратной границей, находим, что

$$
f_{\mathrm{O}}(n ; u)=A_{\mathrm{O}}(2 n-2) \sigma^{2 n^{2}-5 n-2}(a) \varphi(2 n ; u) .
$$

Из этого равенства и соотношения (24) следует, что при $a=e^{i \pi / 3}$ справедливо равенство

$$
\frac{1}{A_{\mathrm{O}}(2 n-2)} \sum_{r=2}^{2 n} A_{\mathrm{O}}(2 n, r) t^{r-2}=\frac{\sigma^{6 n-3}(a) \varphi(2 n ; u)}{\sigma^{2 n-2}(a u) \sigma^{4 n-2}(u) \sigma\left(u^{2}\right)} .
$$

Сравнивая это равенство с (11), приходим к следующей теореме.

ТЕОрема 6. Детальнье перечисления внедиагонально симметричных матрии чередующихся знаков и всех матриц чередующихся знаков связаны равенством

$$
\frac{1}{A_{\mathrm{O}}(2 n-2)} \sum_{r=1}^{2 n} A_{\mathrm{O}}(2 n, r) t^{r-1}=\frac{1}{A(2 n-1)} \frac{t}{t+1} \sum_{r=1}^{2 n} A(2 n, r) t^{r-1}
$$

Сравнивая утверждение этой теоремы с равенством $(23)$ и имея в виду, что $A_{\mathrm{O}}(2)=$ $A_{\mathrm{V}}(3)=1$, получаем

СЛЕДСТВИЕ 5. Детальные перечисления внедиагонально симметричных матрич чередующихся знаков и вертикально симметричных матрии чередующихся знаков совпадают: $A_{\mathrm{O}}(2 n, r)=A_{\mathrm{V}}(2 n+1, r)$.

Это и есть гипотеза, предложенная Кутиным и Юеном.

Отметим, что детерминантные представления, даваемые теоремами 2,3 и 5 , могут быть получены из детерминантных представлений Изергина-Корепина и Куперберга, если воспользоваться равенствами между детерминантами и пфаффианами, полученными Окадой [15].

Благодарности. Работа авторов была частично поддержана Российским фондом фундаментальных исследований (гранты № 01-01-00201 и № 04-01-00352) и INTAS (грант № 00-00561). 


\section{Список литературы}

[1] M. T. Batchelor, J. de Gier, B. Nienhuis. J. Phys. A. 2001. V. 34. P. L265-L270; cond-mat/0101385; Int. J. Mod. Phys. B. 2002. V. 16. P. 1883-1890; math-ph/0204002; P. Di Francesco, P. Zinn-Justin, J.-B. Zuber. A bijection between classes of fully packed loops and plane partitions. math.CO/0311220; J. de Gier, M. Batchelor, B. Nienhuis, S. Mitra. The XXZ spin chain at $\Delta=-1 / 2$ : Bethe roots, symmetric functions and determinants. math-ph/0110011; J. de Gier, B. Nienhuis, P. A. Pearce, V. Rittenberg. J. Stat. Phys. 2004. V. 114. P. 1-35; cond-mat/0301430; N. Kitanine, J. M. Maillet, N. A. Slavnov, V. Terras. J. Phys. A. 2002. V. 35. P. L385-L391; hep-th/0201134; P. A. Pearce, V. Rittenberg, J. de Gier. Critical $Q=1$ Potts model and Temperley-Lieb stochastic processes. cond-mat/0108051; A. V. Razumov, Yu. G. Stroganov. J. Phys. A. 2001. V. 34. P. 3185-3190; cond-mat/0012141; P. 5335-5340; cond-mat/0102247; A. В. Разумов, Ю. Г. Строганов. ТМФ. 2004. T. 138. C. 395-400; math.CO/0104216; A. V. Razumov, Yu. G. Stroganov. $\mathrm{O}(1)$ loop model with different boundary conditions and symmetry classes of alternating-sign matrices. cond-mat/0108103; J.-B. Zuber. On the counting of fully packed loop configurations. Some new conjectures. math-ph/0309057.

[2] Yu. G. Stroganov. A new way to deal with Izergin-Korepin determinant at root of unity. math-ph/0204042.

[3] G. Kuperberg. E-mail message to private "domino" forum, 29 August 2003; for access to forum contact Jim Propp at propp@math.wisc.edu.

[4] G. Kuperberg. Ann. Math. 2002. V. 156. P. 835-866; math.CO/0008184.

[5] V. E. Korepin. Commun. Math. Phys. 1982. V. 86. P. 391-418.

[6] N. Elkies, G. Kuperberg, M. Larsen, J. Propp. J. Algebraic Combin. 1992. V. 1. P. 111-132.

[7] G. Kuperberg. Int. Math. Res. Notices. 1996. V. 3. P. 139-150.

[8] А. Г. Изергин. ДАН СССР. 1987. Т. 297. № 2. С. 331-333; Н. М. Боголюбов, А. Г. Изергин, B. E. Корепин. Корреляционные функции интегрируемых систем и квантовый метод обратной задачи. М.: Наука, 1992.

[9] W. H. Mills, D. P. Robbins, H. Rumsey. Invent. Math. 1982. V. 66. P. 73-87; J. Combin. Theory. Ser. A. 1983. V. 34. P. 340-359.

[10] D. Zeilberger. Electron. J. Combin. 1996. V. 3. № 2. P. \#R13; math.CO/9407211.

[11] O. Tsuchiya. J. Math. Phys. 1998. V. 39. P. 5946-5951.

[12] D. Zeilberger. New York J. Math. 1996. V. 2. P. 59-68; math.CO/9606224.

[13] Yu. G. Stroganov. J. Phys. A. 2001. V. 34. P. L179-L185.

[14] D. P. Robbins. Symmetry classes of alternating sign matrices. math.CO/0008045.

[15] S. Okada. J. Algebra. 1998. V. 205. P. 337-367. 